\title{
Tight control of blood pressure in pregnant women with nonsevere hypertension: expectations for decreasing adverse maternal and fetal pregnancy outcomes
}

\author{
Akihide Ohkuchi ${ }^{1} \cdot$ Atsuhiro Ichihara $^{2}$ \\ Received: 20 January 2022 / Accepted: 24 January 2022 / Published online: 25 February 2022 \\ (c) The Author(s), under exclusive licence to The Japanese Society of Hypertension 2022
}

It has been controversial regarding blood pressure (BP) levels at which antihypertensive drug therapy should be started in women with hypertensive disorders of pregnancy (HDP), which includes chronic hypertension (CH) as well as hypertension occurring at or after 20 weeks of gestation. In addition, the adequate BP level goal following antihypertensive drug therapy in those with HDP has also been controversial. The Japan Society for the Study of Hypertension in Pregnancy (JSSHP) committee previously recommended that antihypertensive drug therapy should be provided for pregnant women with severe hypertension (more than 160/110 $\mathrm{mmHg}$ ), and BP should be maintained at $140-160 / 90-110 \mathrm{mmHg}$ (Fig. 1) [1]. However, considering the recent recommendations of other guidelines $[2,3]$ and the results of the Control of Hypertension in Pregnancy Study (CHIPS) study [4], the JSSHP committee changed the recommendations for the initial BP levels at which to start antihypertensive drugs and the BP level goal following antihypertensive drug therapy in pregnant women with hypertension in the newest best practice guide (2021) [5]: (1) antihypertensive drug therapy should be used in pregnant women with severe hypertension, for whom BP levels equal to or more than $160 / 110 \mathrm{mmHg}$ are repeatedly observed; however, it is possible to start antihypertensive drug therapy in pregnant women with $\mathrm{BP}$ levels that are $\geq 140 / 90 \mathrm{mmHg}$ at the discretion of attending physicians; and (2) the statement for the BP goal following

Akihide Ohkuchi

okuchi@jichi.ac.jp

1 Department of Obstetrics and Gynecology, Jichi Medical University School of Medicine, Tochigi, Japan

2 Department of Endocrinology and Hypertension, Tokyo Women's Medical University, Tokyo, Japan antihypertensive drug therapy in pregnant women with hypertension was retracted.

There have been concerns that tight control of BP in pregnancy might be associated with the occurrence of nonreassuring fetal status (NRFS) and/or fetal growth restriction (FGR). Regarding the association between tight BP control and the occurrence of NRFS, to the best of our knowledge, there have been no reports in English suggesting such an association, although it can be easily estimated that extreme control of BP levels in pregnant women with severe hypertension might lead to a significant reduction in uterine blood flow according to the decreasing BP levels, finally increasing the appearance of NRFS. Regarding the association between tight BP control and the occurrence of FGR, von Dadelszen et al. [6] reported an association between a fall in mean arterial pressure (MAP) and FGR in pregnant women with hypertension. In 45 randomized controlled trials (RCTs) including 3773 women with mild-to-moderate pregnancy hypertension, in which either placebo/no therapy or antihypertensive therapy was administered to controls, a greater mean difference in MAP with antihypertensive therapy was associated with higher incidence rates of small-for-gestational age (SGA) infants as well as lower mean birthweight [6]. However, in a recent meta-analysis assessing the effects of antihypertensive drug therapy for women with mild-tomoderate hypertension during pregnancy [7], including 63 trials with 5909 women, the use of antihypertensive drugs had no effect on the risk of SGA infants (adjusted relative risk: 0.96 ; $95 \%$ confidence interval: $0.78-1.18$ ), suggesting that tight BP control might not have been performed in recent trials. Disappointingly, this meta-analysis did not include the CHIPS trial, where the effects of less-tight versus tight control of hypertension on pregnancy complications were assessed, and a target BP to prevent the outcome was not assessed [7].

Abe et al. [8], for the first time, estimated optimal BP levels for the prevention of severe hypertension in pregnant women with nonsevere hypertension by assessing the 
Previous recommendation by JSSHP

Initial BP levels starting antihypertensive drugs in pregnant women with hypertension

Goal of BP levels in pregnant women with hypertension

Fig. 1 Proposal of initial BP levels for starting antihypertensive drugs and adequate BP level goals in pregnant women with hypertension. Previously, the JSSHP committee recommended that antihypertensive drug therapy should be used in pregnant women with severe hypertension (more than $160 / 110 \mathrm{mmHg}$ ), and their BP should be kept at $140-160 / 90-110 \mathrm{mmHg}$. In this commentary, we proposed novel thresholds for initiating antihypertensive drug therapy and the adequate BP level goals in pregnant women with hypertension. BP blood pressure, JSSHP Japan Society for the Study of Hypertension in Pregnancy

effects of stratifying mean basal or achieved BP levels on adverse maternal and fetal pregnancy outcomes (APOs). They stratified systolic blood pressure (SBP) levels into 5 classes: 120-129, 130-139, 140-149, 150-159, and 160-169 mmHg, whereas they stratified diastolic blood pressure (DBP) levels into 3 classes: 80-89, 90-99, and 100-109 mmHg [8]. For basal BP levels, a significant interaction among basal SBP levels was found in the incidence rates of preeclampsia and placental abruption. For the achieved BP level, a significant interaction among women who achieved an $\mathrm{SBP}<130 \mathrm{mmHg}$ was found in the incidence rate of severe hypertension, whereas there was no reduction of severe hypertension in women who achieved an SBP of $\geq 140 \mathrm{mmHg}$; these results led to their conclusion that BP-lowering treatment aimed at achieving an SBP $<$ $130 \mathrm{mmHg}$, accompanied by careful monitoring of fetal growth, might be recommended to prevent severe hypertension [8]. These results are consistent with the results of the recent CHIPS study, where less-tight control was associated with a significantly higher frequency of severe maternal hypertension [4]; however, in the most recent guideline of the International Society for the Study of Hypertension in Pregnancy (ISSHP), it is recommended that the target BP for antihypertensive therapy should be a DBP of $85 \mathrm{mmHg}$, regardless of the SBP [9]. Therefore, further study is needed to determine the appropriate target $\mathrm{BP}$ for antihypertensive therapy in women with HDP.

This meta-analysis also revealed that BP-lowering treatment in pregnant women with nonsevere hypertension may contribute to several APOs, including severe hypertension, preeclampsia, severe preeclampsia, placental abruption, and preterm birth [8]. In a previous meta-analysis by Abalos et al. [7], the use of antihypertensive drugs was associated with decreased severe hypertension, but it was not associated with preeclampsia, placental abruption, or preterm birth. As discussed by the authors in the present study, one plausible explanation for this discrepancy may have been the inclusion of several large RCTs in which lowdose aspirin (LDA) was prescribed during the study period $[4,10,11]$. Furthermore, the authors pointed out that the intake of antihypertensive drugs prior to enrollment might have influenced the results. A recent report on the effects of BP levels in early pregnancy on pregnancy outcomes by Ueda et al. [12] suggested that an $\mathrm{SBP}<130 \mathrm{mmHg}$ within 14 weeks of gestation reduced the risk of developing earlyonset superimposed preeclampsia in women with $\mathrm{CH}$, although these effects have not been evaluated in a prospective cohort study or RCT.

Recently, it was revealed that the early administration of LDA in women with a high probability score for the occurrence rate of preterm preeclampsia may reduce its incidence rate by almost 60\% (ASPRE trial) [13]; however, surprisingly, administering LDA for pregnant women with $\mathrm{CH}$ was not effective in the subanalysis of the ASPRE trial [14]. In contrast, a preventive effect of LDA on the occurrence of preeclampsia was reported in women with BP levels of $130-139 \mathrm{mmHg} / 80-89 \mathrm{mmHg}$ at or before 16 weeks of gestation [15]. Therefore, an RCT to evaluate the effect of LDA on pregnant women with $\mathrm{CH}$ is strongly needed in the future.

Based on the current study and recent evidence from the CHIPS trial, we propose novel thresholds for initiating antihypertensive drug therapy and the adequate BP level goal in pregnant women with hypertension (Fig. 1). The use of antihypertensive drugs in pregnant women should be started at BP levels at or more than $140 / 90 \mathrm{mmHg}$ at any gestational week, and an adequate BP level goal might be an SBP/DBP $<130 / 85 \mathrm{mmHg}$.

\section{Compliance with ethical standards}

Conflict of interest The author declares no competing interests.

Publisher's note Springer Nature remains neutral with regard to jurisdictional claims in published maps and institutional affiliations.

\section{References}

1. Takagi K, Yamasaki M, Nakamoto O, Saito S, Suzuki H, Seki H, et al. A review of best practice guide 2015 for care and treatment of hypertension in pregnancy. Hyperetens Res Pregnancy. 2015;3: 65-103.

2. Brown MA, Magee LA, Kenny LC, Karumanchi SA, McCarthy FP, Saito S, et al. International Society for the Study of Hypertension in Pregnancy (ISSHP). The hypertensive disorders of pregnancy: ISSHP classification, diagnosis \& management recommendations for international practice. Pregnancy Hypertens. 2018;13:291-310.

3. National Guideline A: National Institute for Health and Care Excellence: Clinical Guidelines. In: Hypertension in pregnancy: 
diagnosis and management (ng133). edn. London: National Institute for Health and Care Excellence (UK). https://www.nice.org.uk/guida nce/ng133/resources/hypertension-in-pregnancy-diagnosis-and-ma nagement-pdf-66141717671365. Accessed 18 Jan 2022.

4. Magee LA, von Dadelszen P, Rey E, Ross S, Asztalos E, Murphy $\mathrm{KE}$, et al. Less-tight versus tight control of hypertension in pregnancy. N Engl J Med. 2015;372:407-17.

5. Japan Society for the Study of Hypertension in Pregnancy Ed. Best practice guide 2021 for care and treatment of hypertension in pregnancy. Tokyo, Japan: Medical View Co., Ltd.: 2021, 70-72.

6. von Dadelszen P, Ornstein MP, Bull SB, Logan AG, Koren G, Magee LA. Fall in mean arterial pressure and fetal growth restriction in pregnancy hypertension: a meta-analysis. Lancet. 2000;355:87-92.

7. Abalos E, Duley L, Steyn DW, Gialdini C. Antihypertensive drug therapy for mild to moderate hypertension during pregnancy. Cochrane Database Syst Rev. 2018;10:CD002252.

8. Abe M, Arima H, Yoshida Y, Fukami A, Sakima A, Metoki H, et al. Optimal blood pressure target to prevent severe hypertension in pregnancy: a systematic review and meta-analysis. Hypertens Res. 2022. https://doi.org/10.1038/s41440-022-00853-z. Online ahead of print.

9. Magee LA, Brown MA, Hall DR, Gupte S, Hennessy A, Karumanchi SA, et al. The 2021 International Society for the Study of Hypertension in Pregnancy classification, diagnosis \& management recommendations for international practice. Pregnancy Hypertens. 2021;27:148-69.
10. Rezk M, Emarh M, Masood A, Dawood R, El-Shamy E, Gamal A, et al. Methyldopa versus labetalol or no medication for treatment of mild and moderate chronic hypertension during pregnancy: a randomized clinical trial. Hypertens Pregnancy. 2020;39:393-8.

11. Salama M, Rezk M, Gaber W, Hamza H, Marawan H, Gamal A, et al. Methyldopa versus nifedipine or no medication for treatment of chronic hypertension during pregnancy: a multicenter randomized clinical trial. Pregnancy Hypertens. 2019;17:54-8.

12. Ueda A, Hasegawa M, Matsumura N, Sato H, Kosaka K, Abiko K, et al. Lower systolic blood pressure levels in early pregnancy are associated with a decreased risk of early-onset superimposed preeclampsia in women with chronic hypertension: a multicenter retrospective study. Hypertens Res. 2022;45:135-45.

13. Rolnik DL, Wright D, Poon LC, O'Gorman N, Syngelaki A, de Paco Matallana $\mathrm{C}$, et al. Aspirin versus placebo in pregnancies at high risk for preterm preeclampsia. N. Engl J Med. 2017;377:613-22.

14. Poon LC, Wright D, Rolnik DL, Syngelaki A, Delgado JL, Tsokaki T, et al. Aspirin for Evidence-Based Preeclampsia Prevention trial: effect of aspirin in prevention of preterm preeclampsia in subgroups of women according to their characteristics and medical and obstetrical history. Am J Obstet Gynecol. 2017;217:585.e1-5.

15. Huai J, Lin L, Juan J, Chen J, Li B, Zhu Y, et al. Preventive effect of aspirin on preeclampsia in high-risk pregnant women with stage 1 hypertension. J Clin Hypertens. 2021;23:1060-7. 